Draft VERSion SEPTEMBER 13, 2018

Preprint typeset using $\mathrm{L}^{A} \mathrm{~T} \mathrm{E}$ X style emulateapj v. 04/17/13

\title{
STRANGE QUARK STARS AS PROBE OF DARK MATTER
}

\author{
HaO Zheng ${ }^{1}$, And Lie-Wen Chen*1,2 \\ ${ }^{1}$ Department of Physics and Astronomy and Shanghai Key Laboratory for Particle Physics and Cosmology, Shanghai Jiao Tong \\ University, Shanghai 200240, China and \\ ${ }^{2}$ Center of Theoretical Nuclear Physics, National Laboratory of Heavy Ion Accelerator, Lanzhou 730000, China \\ Draft version September 13, 2018
}

\begin{abstract}
We demonstrate that the observation of old strange quark stars (SQSs) can set important limits on the scattering cross sections $\sigma_{q}$ between the light quarks and the non-interacting scalar dark matter (DM). By analyzing a set of 1403 of solitary pulsarlike compact stars in the Milky Way, we find the old solitary pulsar PSR J1801-0857D can set the most stringent upper limits on $\sigma_{q}$ or the DM-proton scattering cross sections $\sigma_{p}$. By converting $\sigma_{q}$ into $\sigma_{p}$ based on effective operator analyses, we show the resulting $\sigma_{p}$ limit by assuming PSR J1801-0857D to be a SQS could be comparable with that of the current direct detection experiments but much weaker (by several orders of magnitude) than that obtained by assuming PSR J1801-0857D to be a neutron star (NS), which requires an extremely small $\sigma_{p}$ far beyond the limits of direct detection experiments. Our findings imply that the old pulsars are favored to be SQSs rather than NSs if the scalar DM were observed by future terrestrial experiments. Subject headings: dark matter - stars: neutron - dense matter - equation of state - astroparticle physics
\end{abstract}

\section{INTRODUCTION}

Observation of the pulsarlike old compact stars can provide constraints on dark matter (DM). Being accreted onto the star by strong gravity, DM can accumulate efficiently inside the star via scattering with the star matter and eventually may collapse into a star-killing black hole $(\mathrm{BH})$. To prevent destruction of the star, the interactions between DM and the star matter must be extremely weak. After the pioneering work by Goldman and Nussinov (Goldman \& Nussinov 1989) on this subject, many works have been devoted to constraining the properties of DM based on the observation of old compact stars (Bertone \& Fairbairn 2008; Lavallaz \& Fairbairn 2010; Kouvaris \& Tinvakov 2011; Kouvaris 2012; McDermott et al. 2012; Bramante et al. 2013, 2014; Bramante \& Linden 2014; Zheng et al. 2015). Among these works, the host compact stars are generally assumed to be neutron stars (NSs), and thus numerous constraints on DM-nucleon interactions have been obtained. In particular, for bosonic DM which is favored by many theories beyond the standard model, if its self-interactions can be ignored, the formation of Bose-Einstein condensate (BEC) state could further facilitate the occurrence of DM collapsing into $\mathrm{BH}$, and the resulting limits on the DMnucleon interactions become far beyond the terrestrial experiments (Bernabei et al. 2008; Aprile et al. 2012; Akerib et al. 2014; Agnese \& Ahmed et al. 2013; Agnese et al. 2014; Aprile et al. 2015; Billard et al. 2014), leading to a conclusion that the bosonic DM cannot be detected directly if the old compact stars are NSs.

However, the composition of pulsarlike compact stars remains unclear and its quest is a big challenge in contemporary science $(\mathrm{Xu}$ 2003; Lattimer \& Prakash 2004; Alford et al. 2007; Weber et al. 2009). Besides the conventional NSs, one important candidate is strange

\footnotetext{
* Corresponding author (email: lwchen@sjtu.edu.cn)
}

quark stars (SQSs) (Itoh 1970; Alford et al. 2007; Weber et al. 2009), made purely of deconfined $u, d$, and $s$ quark matter (with some leptons), i.e., strange quark matter (SQM) which might be the true ground state of QCD matter and is absolutely stable according to the Bodmer-Witten-Terazawa hypothesis (Witten 1984; Weber et al. 2009). Many probes have been proposed to distinguish SQSs from NSs, e.g., SQSs have much larger dissipation rate of radial vibrations (Wang \& Lu 1984) and higher bulk viscosity (Haensel et al. 1989), the spin rate of SQSs can be much closer to the Kepler limit than that of NSs (Madsen 1992), SQSs may cool more rapidly than NSs within the first 30 years (Schaab et al. 1997), the gravity-mode (g-mode) eigenfrequencies in SQSs are much lower than those in NSs (Fu et al. 2008), and so on. In the present work, we show that SQSs could be a good probe of the interactions between light quarks and DM, and the observation of scalar bosonic DM by future terrestrial experiments would favor old pulsars to be SQSs rather than NSs.

This paper is organized as follows. We first briefly introduce the methods of calculating the accretion mass of DM by a compact star in Sec. 2. Then the compact star models are described in Sec. 3. In Sec. 4, we present the results and discussions. Finally, the conclusions are given in $\mathrm{Sec}$. 5

\section{ACCRETION OF DM}

Following our recent work (Zheng et al. 2015), we adopt a spherically symmetric accretion scenario to calculate the capture rate of DM by a structured compact star, and the total mass of DM captured by the star during a time period of $t$ can be obtained as

$$
\begin{aligned}
M_{t}= & 4.07 \times 10^{40} \mathrm{GeV} \frac{M_{S} R_{S}}{1-2.964 \frac{M_{S}}{R_{S}}}\left(\frac{m_{\chi} n_{\chi}}{0.3 \mathrm{GeV} / \mathrm{cm}^{3}}\right) \\
& \times\left(\frac{v_{0}}{220 \mathrm{~km} / \mathrm{s}}\right)^{-1}\left(\frac{t}{\mathrm{Gyr}}\right) f,
\end{aligned}
$$


where $M_{S}$ (in unit of solar mass $M_{\odot}$ ) and $R_{S}$ (in unit of $k m$ ) are the star's mass and radius, respectively; $m_{\chi}$ $\left(n_{\chi}\right)$ is the mass (number density) of DM particles which are assumed to follow a Maxwell-Boltzmann distribution with the mode speed of $v_{0} ; f$ is the fraction of DM particles that undergo at least one collision inside the star and it can be expressed as

$$
f=\left\langle 1-e^{-\sum_{i} \int \sigma_{i} \xi_{i}(r) n_{i}(r) \mathrm{d} l}\right\rangle,
$$

with $\sigma_{i}$ denoting the scattering cross section between $\mathrm{DM}$ and star constituent particle $i$ in free space, $\xi_{i}(r)$ the medium corrections due to the Pauli blocking effect and Fermi motion, and the integration in the exponent is taken over the arc length along the trajectory (l) of DM crossing through the star and the summation is for various constituent particles of the star. Finally, all the possible trajectories of DM inside the compact star are averaged (denoted by the angle brackets) in Eq. (2). The DM-lepton interactions play minor roles and are neglected in the calculations.

After being captured by a compact star, the DM may become thermalized soon with the star matter and gather at the host star center with a radius of about several meters within a typical time period of $t_{\mathrm{th}} \sim 0.2 \mathrm{yr}\left(\frac{m_{\chi}}{\mathrm{TeV}}\right)^{2}\left(\frac{\sigma_{b_{i}}}{10^{-43} \mathrm{~cm}^{2}}\right)^{-1}\left(\frac{T_{c}}{T_{5}}\right)^{-1}$, where $T_{c}$ is the central temperature of the star and $T_{5}=$ $10^{5} \mathrm{~K}$ (McDermott et al. 2012; Bertoni et al. 2013; Bramante et al. 2013; Zheng et al. 2015). Especially, for bosonic DM, the BEC state, confined by the star's gravitational field, can be formed when the number of accumulated DM particles exceeds a critical value of $N_{\mathrm{BEC}} \sim 2 \times 10^{35}\left(T_{c} / T_{5}\right)^{3}$. The DM particles exceeding $N_{\text {BEC }}$ will fall into the ground state and gather within a tiny sphere with a radius $r_{\mathrm{BEC}} \sim 1.4 m_{\chi}^{-1 / 2} \mu \mathrm{m}$, where $m_{\chi}$ is in unit of $\mathrm{GeV}$. The DM in the BEC phase will quickly become self-gravitating and form a boson star inside the host star.

As long as the boson star mass exceeds its Chandrasekhar limit, i.e., $M_{t}>M_{\text {chan }}+m_{\chi} N_{\text {BEC }}$, it collapses into a $\mathrm{BH}$. For non-interacting bosonic DM, we have $M_{\text {chan }}=(2 / \pi) M_{\mathrm{pl}}^{2} / m_{\chi}$ with the Planck mass $M_{\mathrm{pl}}=$ $1.22 \times 10^{19} \mathrm{GeV}$. The newly born BH might grow up and eventually swallow the host star if it accretes the star matter faster than its evaporation through Hawking radiation (see, e.g., Eq. (47) in Ref. (Zheng et al. 2015)). Specifically, we adopt a spherically symmetric Bondi accretion scenario (Shapiro \& Teukolsky 1983) to calculate the accretion rate of star matter, and the $\mathrm{BH}$ is assumed to evaporate through Hawking radiation of photons. The stable growth of $\mathrm{BH}$ mass $M_{\mathrm{BH}}$ is guaranteed by the condition $\left.\frac{\mathrm{d} M_{\mathrm{BH}}}{\mathrm{d} t}\right|_{t=0}>0$. As a result, the observation of old compact stars implies that either $M_{\mathrm{t}}<M_{\text {chan }}+m_{\chi} N_{\mathrm{BEC}}$ or $\left.\frac{\mathrm{d} M_{\mathrm{BH}}}{\mathrm{d} t}\right|_{t=0}<0$ must be satisfied to prevent destruction of these stars. This means that the interactions between DM and star matter cannot be too strong so as to prevent the $\mathrm{BH}$ formation or stable growth of $\mathrm{BH}$ mass, which would put limits on the scattering cross sections between DM and compact star matter.

\section{COMPACT STAR MODELS}

In the present work, SQSs are assumed to be static and consist of neutrino-free SQM in $\beta$-equilibrium with electric charge neutrality. The equation of state (EOS) of SQM is taken from the MIT bag model (Chodos et al. 1974) with two light flavors, i.e., the $u$ and $d$ quarks, and one massive flavor, corresponding to the $s$ quark. We further consider corrections in the thermodynamic potential density due to perturbation theory to first order in $\alpha_{s}$ in the $\overline{\mathrm{MS}}$ scheme (Fraga \& Romatschke 2005; Kurkela et al. 2010; Xu et al. 2015). We select the QCD scale parameter $\Lambda_{\overline{\mathrm{MS}}}$ and the invariant mass parameter $\hat{m}_{s}$ to be $146.2 \mathrm{MeV}$ and $279.9 \mathrm{MeV}$, respectively, according to Table. 1 in ( $\mathrm{Xu}$ et al. 2015). Then the QCD coupling and the $s$ quark mass are determined by the running renormalization subtraction point $\Lambda$ given by

$$
\Lambda=\frac{2}{3}\left(\mu_{u}+\mu_{d}+\mu_{s}\right),
$$

where $\mu_{q}(q=u, d$ and $s)$ denotes the chemical potential of each flavor. The bag constant has been chosen to be $B^{1 / 4}=135.0 \mathrm{MeV}$ to yield a value of $873.6 \mathrm{MeV}$ for the binding energy per baryon for cold SQM in equilibrium versus a value of $954.7 \mathrm{MeV}$ for two-flavor $u$ - $d$ quark matter in equilibrium, and thus the absolutely stable condition is satisfied. The SQS structure is then obtained by solving the Tolman-Oppenheimer-Volkoff equations. Shown in Fig. 1 is the radial density distribution of each quark flavor for a typical SQS with mass $M_{S}=1.4 M_{\odot}$, and the SQS radius is obtained as $R_{S}=11.47 \mathrm{~km}$. Based on Fig. 1, the $f$ in Eq. (2) can then be evaluated. We note that using the MIT bag model without perturbation corrections for the quark interactions (Fu et al. 2008) does not change our conclusions.

For NSs, we adopt the conventional neutron star model in which the NS is assumed to consist of $\beta$-stable and electrically neutral npe $\mu$ matter and the EOS is taken from Skyrme-Hartree-Fock approach using the MSL1 interaction (Zhang \& Chen 2013), and the details can be found in Ref. (Zheng et al. 2015).

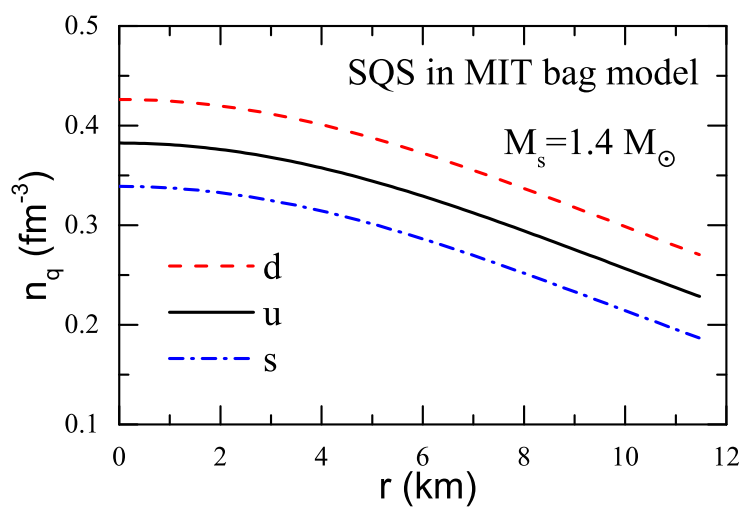

FIG. 1.- (Color online) Radial quark number density distribution for a SQS with mass of $1.4 M_{\odot}$ from the MIT bag model.

The thermalization and BEC formation of DM in the interior of compact stars depend on significantly the star's internal temperature $T_{c}$ which is largely uncertain in observations. For NSs, following our previous work (Zheng et al. 2015), the $T_{c}$ can be estimated from the relatively well-studied NS's surface temperature $T_{s}$ 
based on the study of thermal structure of the insulating envelope presented by Gudmundsson et al. (1982). In particular, Gudmundsson et al. (1982) found that the temperature $T_{b}$ measured at inner boundary of the envelope (with density $\rho \sim 10^{10} \mathrm{~g} \mathrm{~cm}^{-3}$ ) can be related to the surface temperature $T_{s}$ as

$$
T_{b}=1.288 \times 10^{8} \mathrm{~K}\left[\frac{10^{14} \mathrm{~cm} / \mathrm{s}^{2}}{g_{s}}\left(\frac{T_{s}}{10^{6} \mathrm{~K}}\right)^{4}\right]^{0.455},
$$

where $g_{s}$ is the surface gravity. Since old compact stars are already isothermal in their interiors, the $T_{c}$ can be assumed to be equal to $T_{b}$.

For SQSs, contrary to a bare surface with a steep density drop over a few $f m$, they may be wrapped up either in a tiny crust consisting of "normal" matter, i.e., ions and electrons, with maximum density below the neutron drip density (Alcock et al. 1986), or in a heterogeneous (solid) crust made of strange nuggets and electrons (Jaikumar et al. 2006). The former crust is supported by strong electric fields near the surface and has similar properties with the thermal envelope in Gudmundsson's model. Thus it plays the role of an insulating layer and in this case we assume the same relationship between $T_{c}$ and $T_{s}$ as shown in Eq. (4), which leads to an estimate of $T_{c} / T_{s} \simeq 15.1 \times\left(T_{s} / T_{5}\right)^{0.82}$ based on the SQS structure shown in Fig. 1] By assuming $T_{s} \sim T_{5}$, our estimate is nicely consistent with previous assumption presented by Blaschke et al. (2000) and Horvath et al. (1991) with $T_{c} / T_{s}=20$ in their work. For the heterogeneous crust within the strange nuggets model, Jaikumar et al. (2006) found a relatively large crust with radial extent $\Delta R \simeq 40 \mathrm{~m}$ and maximum density up to $\rho \sim 10^{13} \mathrm{~g} \mathrm{~cm}^{-3}$. The opacity of the nuggets matter has two origins, one due to scattering of electrons off nuggets, and the other due to scattering among different electrons. Since the nuggets phase resembles the mixed phase of nuclei and electrons in the crust of normal neutron stars (Jaikumar et al. 2006; Page et al. 2004), thus we can also apply Eq. (4) to estimate $T_{c}$ from $T_{s}$. It should be noted that crust consisting of strange nuggets may exist only if the surface tension between the zero-pressure surface and the vacuum satisfies condition $\sigma<\sigma_{\text {crit }}$ Jaikumar et al. 2006). For the MIT bag model in this work, we have $\sigma \approx 4.1 \mathrm{MeV} \cdot \mathrm{fm}^{-2}$ and $\sigma_{\text {crit }} \approx 135 \mathrm{MeV} \cdot \mathrm{fm}^{-2}$, implying that a crust of mixed phase is indeed favored in our model. The above discussions suggest that Eq. (4) provides a reasonable approach to estimate $T_{c}$ from $T_{s}$ for both NSs and SQSs.

Furthermore, for solitary compact stars older than several million years, the $T_{s}$ is estimated to be lower than $10^{5} \mathrm{~K}$ (Yakovlev \& Pethick 2004; Negreiros et al. 2012). In the present work, we assume a fixed $T_{s}=10^{5} \mathrm{~K}$ for both NSs and SQSs. Since the DM particles accumulated in old compact stars (e.g., those with age older than $10^{9}$ years that we are interested in) essentially come from the accretion process at the late stage of thermal evolution when the $T_{s}$ is lower than $10^{5} \mathrm{~K}$, the resulting constraints in the present work are thus expected to be conservative as a lower $T_{s}$ will lead to stronger constraints.

\section{LIMITS ON $\sigma_{Q}$ AND $\sigma_{P}$}

Since numerous pulsarlike compact stars have been observed in the Milky Way with various states, the resulting constraints from different stars on DM-quark (proton) spin-independent (SI) scattering cross sections $\sigma_{q}$ $\left(\sigma_{p}\right)$ should vary from one star to another. We thus scan over all the available solitary compact objects and finally figure out the one leading to the most stringent limits. Particularly, we only focus on the isolated pulsar systems to avoid the additional complexity due to the evolution history of pulsars in a binary (or more complex) system. Moreover, the pulsars with age less than 1 million years are ignored since they are expected to have little time to accrete enough DM and to have a relatively higher temperature than the older ones. Whereas there are little information on the masses and radii of the solitary pulsars, we assume all of them have the fiducial mass of $1.4 M_{\odot}$ with the radii calculated from the NS or SQS models. From Eq. (10), for compact stars with the same structure, the variation of the constraints from different compact stars is mainly due to the term $w_{\chi}(r)=\rho_{\chi}(r) \cdot t$. Here the living age $t$ is taken as the pulsar's spindown age. The DM mass density $\rho_{\chi}(r)=m_{\chi} n_{\chi}(r)$ depends on the halo model for which we adopt here the spherically symmetric generalized Navarro-Frenk-White (NFW) profile (Navarro et al. 1996) and Einasto profile (Merritt et al. 2006), i.e.,

$$
\rho_{\chi}(r)= \begin{cases}\frac{\bar{\rho}_{s}}{\left(1+\frac{r}{r_{s}}\right)^{3-\alpha}\left(\frac{r}{r_{s}}\right)^{\alpha}} & (\mathrm{NFW}), \\ \bar{\rho}_{s} e^{-\frac{2}{\alpha}\left[\left(\frac{r}{r_{s}}\right)^{\alpha}-1\right]} & \text { (Einasto) }\end{cases}
$$

where $r_{s}$ is the scale radius, $\bar{\rho}_{s}$ is the scale density and $\alpha$ is the inner slope for the NFW profile and a shape parameter for the Einasto profile. We take $\alpha=1(0.17)$ for NFW (Einasto) based on the results of N-body simulations (Navarro et al. 2010) and $r_{s}=20 \mathrm{kpc}$ (Iocco et al. 2011). The $\bar{\rho}_{s}$ is then obtained by fitting the solar system DM density $\rho_{0}=0.4 \mathrm{GeV} / \mathrm{cm}^{3}$. We scan over all the available 1403 solitary pulsars recorded in the ATNF Pulsar Catalogue (Manchester et al. 2005) and find the one maximizing $w_{\chi}(r)$ is PSR J1801-0857D, a solitary pulsar with age of $9.71 \mathrm{Gyr}$ and distance of $3.06 \mathrm{kpc}$ from the galactic center. The corresponding $w_{\chi}(r)$ is $16.0(19.1) \mathrm{GeV} \cdot \mathrm{Gyr} \cdot \mathrm{cm}^{-3}$ for NFW (Einasto), indicating small model dependence on the halo profiles for our results. It should be emphasized that, within the present framework, PSR J1801-0857D can set the strongest constraints on DM-quark and DM-proton scattering cross sections among all the 1403 pulsars. All results in the following are calculated by using parameters of PSR J1801$0857 \mathrm{D}$.

Now we can directly constrain $\sigma_{u}\left(\sigma_{p}\right)$ from the existence of PSR J1801-0857D by assuming it is a SQS (NS). Furthermore, one can convert the limits on $\sigma_{u}$ obtained from the SQS assumption to those on $\sigma_{p}$, and then compare with the constraints obtained from the NS assumption as well as the results released by various direct detection experiments. Based on general operator analyses, for scalar DM, the effective operators describing the DM-quark interactions that can generate DMnucleon SI scattering are limited to the following two 
classes (Gao et al. 2013):

$$
a_{q} \phi^{\dagger} \phi \bar{q} q, \quad b_{q} \phi^{\dagger} \overleftrightarrow{\partial}^{\mu} \phi \bar{q} \gamma_{\mu} q
$$

The above first and second operators lead to the scalar and vector DM-quark interactions, respectively, with $a_{q}$ and $b_{q}$ being the coupling coefficients. The corresponding effective operators describing the DM-nucleon interactions are $f_{N}^{s} \phi^{\dagger} \phi \bar{N} N$ and $f_{N}^{v} \phi^{\dagger} \overleftrightarrow{\partial} \mu \phi \bar{N} \gamma_{\mu} N$, respectively, where $N$ denotes protons $(p)$ or neutrons $(n)$ and $f_{N}$ is related to $a_{q}$ and $b_{q}$ by

$$
f_{N}^{s}=\frac{1}{2 m_{\chi}} \sum_{q} B_{q}^{N} a_{q}, f_{N}^{v}=\sum_{q} B_{q}^{N} b_{q}
$$

with the dimensionless quantities (Gao et al. 2013) $B_{u}^{p}=9.3, B_{u}^{n}=6.5, B_{d}^{p}=5.1, B_{d}^{n}=7.1$ and $B_{s}^{p, n}=1.2$ for scalar interaction, and $B_{u}^{p}=2, B_{u}^{n}=1, B_{d}^{p}=1$, $B_{d}^{n}=2$ and $B_{s}^{p, n}=0$ for vector interaction. Eq. (7) does not include the contributions of sea quarks and gluons to $f_{N}^{s}$, which can be effectively encoded in an additional free coefficient (Cirigliano et al. 2014) and will be discussed later. Then the DM-proton scattering cross section, for both scalar and vector interactions, takes the form $\sigma_{p}=\left(\mu_{p}^{2} / \pi\right) f_{p}^{2}$ while the DM-quark scattering cross sections are given by $\sigma_{q}^{s}=(1 / 4 \pi)\left(\mu_{q}^{2} a_{q}^{2} / m_{\chi}^{2}\right)$ for scalar interaction and $\sigma_{q}^{v}=\mu_{q}^{2} b_{q}^{2} / \pi$ for vector interaction. Here $\mu_{p}\left(\mu_{q}\right)$ is the DM-proton (DM-quark) reduced mass. In the present work, the current masses of various quark flavors are taken as $m_{u}=2.3 \mathrm{MeV}, m_{d}=4.8 \mathrm{MeV}$, and $m_{s}=95 \mathrm{MeV}$. Thus one can derive limits on $\sigma_{p}$ from those on $\sigma_{q}$ with a specific type of DM-quark interaction.

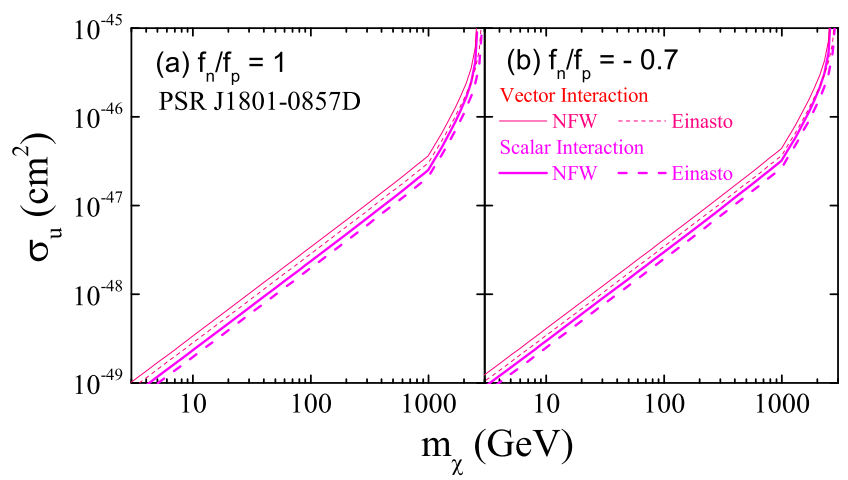

FIG. 2.- (Color online) Limits in the $m_{\chi}-\sigma_{u}$ plane for scalar DM by assuming PSR J1801-0857D to be a SQS. DM is assumed to only interact with the first family of quarks.

Shown in Fig. 2 are the limits on $\sigma_{u}$ vs $m_{\chi}$ by assuming that DM only interact with the first family of quarks for two cases of the so-called isospin-violating DM (Feng et al. 2011) with $f_{n} / f_{p}=1$ and -0.7 . Note that the cut-off mass around several TeV in Fig. 22 implies that PSR J1801-0857D fails to put constraints on heavier DM since for them Hawking radiation will always overwhelm the $\mathrm{BH}$ accretion and so the $\mathrm{BH}$ cannot grow up stably. In addition, $B_{u}^{N}$ and $B_{d}^{N}$ are different for scalar and vector interactions, and so the limits show an interaction dependence with a stronger limit from the scalar interaction. On the other hand, the limits with NFW and
Einasto only display a very small difference as expected. From Fig. 2] one can see that PSR J1801-0857D indeed can put extremely strong limits on $\sigma_{u}$, especially for light $\mathrm{DM}$, at the order of $10^{-49} \mathrm{~cm}^{2}$. Since similar results on $\sigma_{d}$ can be obtained via relationship $\sigma_{d} / \sigma_{u}=\left(\mu_{d} / \mu_{u}\right)^{2} g_{d u}^{2}$ where $g_{d u}=a_{d} / a_{u}\left(b_{d} / b_{u}\right)$ for scalar (vector) interaction is related to the isospin-violating factor $g_{n p}=f_{n} / f_{p}$ by $g_{d u}=\left(g_{n p} B_{u}^{p}-B_{u}^{n}\right) /\left(B_{d}^{n}-g_{n p} B_{d}^{p}\right)$, our present results are potentially useful in constraining various model parameters for DM-quark interactions (Gao et al. 2013).

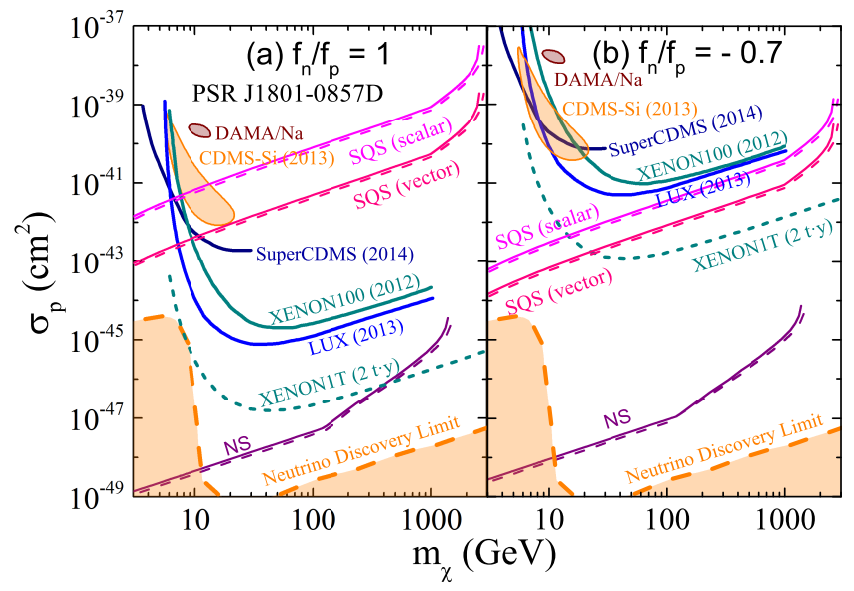

Fig. 3.- (Color online) Limits in the $m_{\chi}-\sigma_{p}$ plane for scalar DM by assuming PSR J1801-0857D to be a SQS or a NS with NFW (solid lines) and Einasto (dashed lines) DM halo profile. For the SQS case, DM is assumed to only interact with the first family of quarks. The corresponding results from direct detection experiments, i.e., DAMA-Libra (Bernabei et al. 2008), CDMS-II(Si) (Agnese \& Ahmed et al. 2013), XENON100 (Aprile et al. 2012), LUX (Akerib et al. 2014), SuperCDMS(Ge) (Agnese et al. 2014) as well as the future XENON1T (Aprile et al. 2015) and the "neutrino discovery limit" (Billard et al. 2014) are also included for comparison.

Then we move to the $m_{\chi^{-}} \sigma_{p}$ plane. Shown in Fig. 3 are the constraints on $\sigma_{p}$ either obtained directly from the NS assumption or derived from the $\sigma_{u}$ as shown in Fig. 2 from the SQS assumption. It is very interesting to see that, for both cases of $f_{n} / f_{p}=1$ and -0.7 , the limits on $\sigma_{p}$ derived from $\sigma_{u}$ are shifted upward dramatically compared with that on $\sigma_{u}$ and become significantly larger than the limits given by NS. This is due to the fact that the $\sigma_{p}$ converted from the $\sigma_{q}$ is enlarged by a factor of $\left(\mu_{p} / \mu_{q}\right)^{2}\left(B_{u}^{p}+g_{d u} B_{d}^{p}\right)^{2}$ with an amplitude of about $10^{6}-10^{7}$, and thus the limits on $\sigma_{p}$ set by the SQS assumption are significantly weakened compared with that set by the NS assumption. For comparison, the current limits and regions on $\sigma_{p}$ reported by various direct detection experiments are also shown in Fig. 3. including the regions from DAMA-Libra (Bernabei et al. 2008) and CDMS-Si Agnese \& Ahmed et al. 2013) experiments and the limits from SuperCDMS (Agnese et al. 2014), XENON100 (Aprile et al. 2012) and LUX (Akerib et al. 2014) groups. Also included are the expected sensitivity of future experiment XENON1T Aprile et al. 2015) and the "neutrino discovery limit" (Billard et al. 2014) which sets limit on the sensitivity of the direct detection method.

It is seen from Fig. 3 that, for both cases with $f_{n} / f_{p}=$ 1 and -0.7 , the NS assumption provides the most strin- 
gent constraints. In particular, even for the isospininvariant case that $f_{n} / f_{p}=1$, they are beyond the sensitivity of future XENON1T up to $m_{\chi} \sim 400 \mathrm{GeV}$. However, the constraints set by SQS assumption with scalar interaction become compatible with the CDMS-Si contour for $f_{n}=f_{p}$, and the relatively more stringent constraints from vector interaction are still weaker than that of the current xenon-based experiments. Moreover, for the isospin-violating case with $f_{n} / f_{p}=-0.7$ (i.e., the so-called xenophobic DM), while the tension among various direct detection experiments is largely ameliorated due to the destructive interference of DM scattering with protons and neutrons inside the target nuclei, all the currently favored DM regions are excluded even by the softest limits from the SQS assumption. But it is interesting to see that future XENON1T, even in the xenophobic case, is expected to have higher sensitivity than the limits set by the SQS assumption for massive DM. This means that if future experiments had observed scalar DM signals within the mass region $\gtrsim \mathcal{O}(10) \mathrm{GeV}$, then the old compact objects are favored to be SQSs rather than NSs. On the other hand, if no positive signals were observed by XENON1T, the old compact stars still provide important constraints on models which predict scalar DM with mass lighter than $\sim \mathcal{O}(10) \mathrm{GeV}$. Therefore direct detection experiments for DM provide a novel way to probe the nature of pulsars.

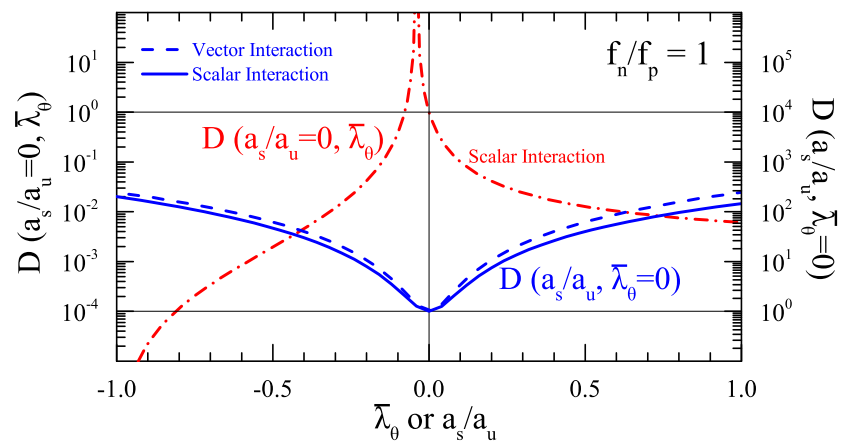

FIG. 4.- (Color online) Degradation factor due to the contributions from $s$ quark and heavy quarks (gluon) for the limits in the $m_{\chi}-\sigma_{p}$ plane by assuming PSR J1801-0857D to be a SQS in the case of $f_{n}=f_{p}$.

Finally, we discuss the effects of $s$ quark and heavy quarks (gluon) by showing in Fig. 4 the so-called degradation factor (Feng et al. 2013) which measures the suppression or amplification effects on the $\sigma_{p}$ constraints and is defined as

$$
D\left(g_{s u}, \bar{\lambda}_{\theta}\right)=\frac{\sigma_{p}(0,0)}{\sigma_{p}\left(g_{s u}, \bar{\lambda}_{\theta}\right)}
$$

with $g_{s u}=a_{s} / a_{u}$ and $\bar{\lambda}_{\theta}$ the rescaled heavy quark (gluonic) coupling defined in Ref. (Cirigliano et al. 2014).
Noting that the isospin-violating effect is very small for large $g_{s u}$ or $\bar{\lambda}_{\theta}$, we thus only show the results with

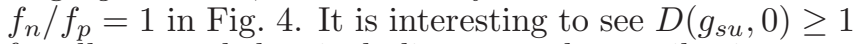
for all $g_{s u}$, and thus including $s$ quark contributions can significantly increase the sensitivity of the $\sigma_{p}$ constraints set by a SQS for both scalar and vector DM-quark interactions. On the other hand, for the heavy quark (gluon) effects with vector interaction, the sea quarks and gluons do not contribute to $f_{p, n}$ due to the conservation of vector current, and thus we show the degradation factor only for scalar interaction in Fig. 4. One can see the heavy quark (gluon) effects can either increase or decrease the sensitivity, depending on the specific value of $\bar{\lambda}_{\theta}$. To further constrain $g_{s u}$ and $\bar{\lambda}_{\theta}$, other independent constraints are necessary, e.g., from the collider experiments (Goodman et al. 2010).

\section{CONCLUSIONS}

For scalar DM with ignorable self-interactions, we have shown that the old strange quark stars can directly put important constraints on DM-quark scattering cross sections $\sigma_{q}$, which can be further converted into the constraints on DM-proton scattering cross sections $\sigma_{p}$ based on effective operator analyses. By analyzing a set of 1403 of solitary pulsarlike compact stars in the Milky Way, we have found that the old pulsar PSR J1801-0857D can put the most stringent constraints on $\sigma_{q}$ and $\sigma_{p}$. Furthermore, we have demonstrated that while the limits on $\sigma_{p}$ obtained by assuming PSR J1801-0857D to be a neutron star essentially rule out the possibility of detecting scalar DM in terrestrial labs through direct detection experiments, the extracted limits from assuming PSR J1801-0857D to be a strange quark star are significantly weakened to be comparable with the terrestrial direct detection experiments. Our results have indicated that DM direct detection experiments provide a novel way to probe the nature of old pulsarlike compact stars, and the old pulsars are favored to be strange quark stars rather than neutron stars if scalar DM could be observed by future direct detection experiments, e.g., XENON1T.

We are grateful to Ren-Xin $\mathrm{Xu}$ for helpful discussions. This work was supported in part by the Major State Basic Research Development Program (973 Program) in China under Contract Nos. 2015CB856904 and 2013CB834405, the NSFC under Grant Nos. 11275125 and 11135011, the "Shu Guang" project supported by Shanghai Municipal Education Commission and Shanghai Education Development Foundation, the Program for Professor of Special Appointment (Eastern Scholar) at Shanghai Institutions of Higher Learning, and the Science and Technology Commission of Shanghai Municipality (11DZ2260700).

\section{REFERENCES}

Agnese, R., Ahmed, Z., Anderson, A. J., et al. 2013, Phys. Rev. Lett., 111, 251301

Agnese, R., Anderson, A. J., Asai, M., et al. 2014, Phys. Rev. Lett., 112, 241302

Akerib, D. S., Araujo, H. M., Bai, X., et al. 2014, Phys. Rev. Lett., 112, 091303

Alcock, C., Farhi, E., \& Olinto, A. 1986, ApJ, 310, 261
Alford, M., Blaschke, D., Drago, A., et al. 2007, Nature (London), 445, E7

Aprile, E., Alfonsi, M., Arisaka, K., et al. 2012, Phys. Rev. Lett., 109, 181301

Aprile, E., Aalbers, J., Agostini, F., et al. 2015, arXiv:1512.07501 Bernabei, R., Belli, P., Cappella, F., et al. 2008, Eur. Phys. J. C, 56,333 
Bertone, G., \& Fairbairn, M. 2008, Phys. Rev. D, 77, 043515

Bertoni, B., Nelson, A. E., \& Reddy, S. 2013, Phys. Rev. D, 88, 123505

Billard, J., Strigari, L., \& Figueroa-Feliciano, E. 2014, Phys. Rev. D, 89, 023524

Blaschke, D., Klahn, T., \& Voskresensky, D. N. 2000, ApJ, 533, 406

Bramante, J., Fukushima, K., \& Kumar, J. 2013, Phys. Rev. D, 87, 055012

Bramante, J., Fukushima, K., Kumar, J., \& Stopnitzky, E. 2014, Phys. Rev. D, 89, 015010

Bramante, J., \& Linden, T. 2014 Phys. Rev. Lett., 113, 191301

Chodos, A., Jaffe, R. L., Johnson, K., Thorne, C. B., \& Weisskopf, V. F. 1974, Phys. Rev. D, 9, 3471

Cirigliano, V., Graesser, M. L., Ovanesyan, G., \& Shoemaker, I. M. 2014, Phys. Lett. B, 739, 293

Farhi, E., \& Jaffe, R. L. 1984, Phys. Rev. D, 30, 2379

Feng, J. L., Kumar, J., Marfatia, D., \& Sanford, D. 2011, Phys. Lett. B, 703, 124-127

Feng, J. L., Kumar, J., \& Sanford, D. 2013, Phys. Rev. D, 88, 015021

Fraga, E. S. \& Romatschke, P. 2005, Phys. Rev. D, 71, 105014

Fu, W.-J., Wei, H.-Q., \& Liu, Y.-X. 2008, Phys. Rev. Lett., 101, 181102

Gao, X., Kang, Z., \& Li, T. 2013, JCAP, 01, 021

Goldman, I., \& Nussinov, S. 1989, Phys. Rev. D, 40, 3221

Goodman, J., Ibe, M., Rajaraman, A., et al. 2010, Phys. Rev. D, 82,116010

Gudmundsson, E. H., Pethick, C. J., \& Epstein, R. I. 1982, ApJ, 259, L19

Haensel, P., Zdunik, J.-L., \& Schaeffer, R. 1989, Astron. Astrophys., 217, 137

Horvath, J., Benvenuto, O., \& Vucetich, H. 1991, Phys. Rev. D, 44, 3797

Iocco, F., Pato, M., Bertonea, G., \& Jetzer, P. 2011, JCAP, 1111, 029

Itoh, N. 1970, Prog. Theor. Phys., 44, 291

Jaikumar, P., Reddy, S., \& Steiner, A. W. 2006, Phys. Rev. Lett., 96, 041101

Kouvaris, C. 2012, Phys. Rev. Lett., 108, 191301
Kouvaris, C., \& Tinyakov, P. 2011, Phys. Rev. Lett., 107, 091301 Kurkela, A., Romatschke, P., \& Vuorinen, A. 2010, Phys. Rev. D 81,105021

Lattimer, J. M., \& Prakash, M. 2004, Science, 304, 536

de Lavallaz, A., \& Fairbairn, M. 2010, Phys. Rev. D, 81, 123521

Lynch, R. S., Ransom, S. M., Freire, P. C. C., \& Stairs, I. H.

2011, ApJ, 734, 89

Madsen, J. 1992, Phys. Rev. D, 46, 3290

Manchester, R. N., Hobbs, G. B., Teoh, A., \& Hobbs, M. 2005 Astron. J. 129, 1993,

http://www.atnf.csiro.au/people/pulsar/psrcat/

McDermott, S. D., Yu, H.-B., \& Zurek, K. M. 2012, Phys. Rev. D, 85, 023519

Merritt, D., Graham, A. W., Moore, B., et al. 2006, Astron. J., 132,2685

Navarro, J. F., Frenk, C. S., \& White, S. D. M. 1996, ApJ, 462, 563

Navarro, J. F., Ludlow, A., Springel, V., et al. 2010, Mon. Not. Roy. Astron. Soc., 402, 21

Negreiros, R., Schramm, S., \& Weber, F. 2012, Phys. Rev. D, 85, 104019

Page, D., Lattimer, J. M., Prakash, M., \& Steiner, A. W. 2004, ApJS, 155, 623

Shapiro, S. L., \& Teukolsky, S. A. 1983, in Black Holes, White Dwarfs, and Neutron Stars: The Physics of Compact Objects (Wiley, New York)

Schaab, C., Hermann, B., Weber, F., \& Weigel, M. K. 1997, ApJ, 480, L111

Wang, Q.-D., \& Lu, T. 1984, Phys. Lett. B, 148, 211

Weber, F., Negreiros, R., \& Rosenfield, P. 2009, in Neutron Stars and Pulsars (Springer, Berlin, Heidelberg)

Witten, E. 1984, Phys. Rev. D, 30, 272

Xu, J.-F., Peng, G.-X., Hou, D.-F., \& Chen, L.-W. 2015, Phys. Rev. D, 92, 025025

Xu, R.-X. 2003, ApJ, 596, L59

Yakovlev, D. G., \& Pethick, C. J. 2004, Annu. Rev. A\&A, 42, 169

Zhang, Z., \& Chen, L. W. 2013, Phys. Lett. B, 726, 234

Zheng, H., Sun, K.-J., \& Chen, L.-W. 2015, ApJ, 800, 141 